13

\title{
Исследования проводящих и сегнетоэлектрических свойств ВZT-пленок
}

\author{
() Е.В. Гущина, Б.Р. Бородин, В.А. Шаров, В.В. Осипов, С.И. Павлов, М.А. Яговкина, М.С. Дунаевский \\ Физико-технический институт им. А.Ф. Иофрфе РАН, \\ 194021 Санкт-Петербург, Россия \\ e-mail: katgushch@yandex.ru
}

Поступило в Редакцию 14 февраля 2020 г.

В окончательной редакции 23 мая 2020 r.

Принято к публикации 5 июня 2020 г.

С помощью контактной проводящей сканирующей зондовой микроскопии и микроскопии пьезоотклика исследованы процессы локальной поляризации в тонких пленках $\mathrm{BaTi}_{1-x} \mathrm{Zr}_{x} \mathrm{O}_{3}$. Установлена связь направления созданных доменов и величины протекающих токов. Найдена величина остаточной поляризации, а с помощью сканирующей зондовой микроскопии измерена петля гистерезиса и определены значения пьезомодуля $d_{z z}$ и значение коэрцитивного поля $E_{c}$ для этих пленок.

Ключевые слова: тонкие сегнетоэлектрические ВZT-пленки, сканирующая зондовая микроскопия, карты распределения токов, сигнал пьезоотклика, петля гистерезиса.

DOI: $10.21883 / J T F .2020 .12 .50137 .52-20$

\section{Введение}

Тонкие сегнетоэлектрические (СЭ) пленки твердых растворов цирконата-титаната бария $\mathrm{Ba}\left(\mathrm{Ti}_{1-x} \mathrm{Zr}_{x}\right) \mathrm{O}_{3}$ (BZT) являются перспективными материалами для создания различных СВЧ-устройств $[1,2]$. Например, пленки BZT $(0.35<\mathrm{x}<0.6)$ нашли применение в перестраиваемых микроволновых устройствах [3]. Кроме того, эти материалы вызывают интерес в связи с тем, что введение в твердый раствор более стабильных атомов $\mathrm{Zr}$ по сравнению с атомами Ті может приводить к уменьшению СВЧ-потерь и токов утечек в пленках [4]. Также на величины токов утечек могут оказывать влияние кристаллическая структура пленки и свойства границ зерен в поликристаллических пленках [5].

Величина и направление вектора остаточной поляризации являются важными параметрами, определяющими эффективность работы устройств памяти на базе BZT. Процесс переключения поляризации проявляется в гистерезисе зависимости величины поляризации от приложенного к пленке напряжения. Этот гистерезис можно измерить с помощью сканирующей зондовой микроскопии (С3М). Так, в работах [6-9] с помощью СЗМ-зонда на верхнем Рt-электроде были измерены свойства и петли гистерезиса сегнетоэлектрических пленок. При исследовании керамических образцов $\mathrm{Ba}\left(\mathrm{Ti}_{1-x} \mathrm{Zr}_{x}\right) \mathrm{O}_{3} \quad(x=0.2-0.23)$ в сигнале пьезоотклика наблюдалась различная доменная структура. Также установлено, что с увеличением содержания $\mathrm{Zr}$ минимальный размер нанодоменов уменьшается, а процесс переключения поляризации становится возможным при более низких прикладываемых электрических полях [6]. Переключение поляризации путем подачи напряжения противоположного знака и последующая регистрация этого переключения с помощью измерения пьезоотклика и петель гистерезиса с помощью СЗМ были продемонстрированы в работах [6-9]. Однако в работах $[4,7]$ изучались поликристаллические BZT-пленки, в работе [6] BZT-керамика, а в работе [8] тонкая эпитаксиальная BZT-пленка с добавлением $\mathrm{Ba}_{0.7} \mathrm{Ca}_{0.3} \mathrm{TiO}_{3}$. В отличие от керамических и поликристаллических образцов в монокристаллических пленках отсутствуют границы зерен, которые могут являться потенциальными барьерами при переносе заряда и, будучи проводящими, увеличивать токи утечки. Поэтому монокристаллические пленки более предпочтительны для использования в устройствах микроэлектроники [10].

Стоит отметить, что процессы переключения поляризации в BZT-пленках с большими концентрациями циркония $(x=0.2,0.35)$ и без каких-либо добавок (оксид железа, кальция) методами СЗМ исследованы недостаточно полно. Таким образом, в настоящей работе решались следующие задачи:

1) определение состава и ориентации BZT-пленок, установление текстуры (поликристаллические или монокристаллические) пленки, измерение величины остаточной поляризации, диэлектрической проницаемости, диэлектрических потерь;

2) исследование процесса переключения СЭ доменов в BZT пленках $(x=0.2,0.35)$ методами С3М;

3) выявление взамосвязи величин протекающих токов с направлением поляризации в этих пленках;

4) измерение петель СЭ гистерезиса и определение значений пьезомодуля $d_{33}$ и коэрцитивных полей $E_{c}$.

\section{1. Образцы и методы}

Исследуемые однослойные и многослойные
$\mathrm{C}$ 
(\#2) $\quad \mathrm{BaTi}_{0.65} \mathrm{Zr}_{0.35} \mathrm{O}_{3}(40 \mathrm{~nm}) \quad / \mathrm{BaTi}_{0.8} \mathrm{Zr}_{0.2} \mathrm{O}_{3} \quad(20 \mathrm{~nm})$ $/ \mathrm{BaTi}_{0.65} \mathrm{Zr}_{0.35} \mathrm{O}_{3}(40 \mathrm{~nm})$, (\#3) $\mathrm{BaTi}_{0.8} \mathrm{Zr}_{0.2} \mathrm{O}_{3}(100 \mathrm{~nm})$ осаждались методом высокочастотного распыления на подложках $\mathrm{Si} / \mathrm{SrTiO}_{3}$. Электроды $\mathrm{SrRuO}_{3}$ наносились импульсным лазерным осаждением [9]. Выбор исследуемых образцов был обусловлен следующими обстоятельствами. Известно, что с увеличением концентрации $\mathrm{Zr}$ происходит существенное снижение температуры Кюри ВZT-пленок [11], что позволяет управлять величиной диэлектрической проницаемости BZT. При концентрации $X_{\mathrm{Zr}}=0.2$ (\#3) температура Кюри выше комнатной, а при $X_{\mathrm{Zr}}=0.35$ (\#1) - близка к комнатной. Это означает, что пьезоэлектрические свойства образца с $X_{\mathrm{Zr}}=0.35$ могут быть несколько ослаблены за счет присутствия несегнетоэлектрической фазы. Образец с чередующимся составом был добавлен как промежуточный по составу между образцами (\#1) и (\#3). Кроме того, в работе [12] было показано, что применение пленок с чередующимися по составу слоями позволяет снизить токи утечек.

Для комплексного исследования свойств данных пленок в работе использовались следующие методы и методики:

1. Методы контактной проводящей сканирующей зондовой микроскопии (к-СЗМ) и микроскопии пьезоотклика. Использование определенной конфигурации СЗМ микроскопа для измерения сверхмалых токов позволяет фиксировать токи с точностью до $0.02 \mathrm{pA}$. С помощью микроскопии пьезоотклика были измерены петли СЭ гистерезиса [13] и определены значения пьезоэлектрического модуля $d_{33}$. Все зондовые измерения выполнялись с помощью СЗМ-микроскопов „SOLVER P47-PRO“ (NTMDT) и „NTegra-Aura“. Использовались зонды с проводящим алмазным покрытием DCP-11 (NT-MDT).

2. Рентгенодифракционные исследования проводились на дифрактометре для анализа поликристаллов „D2Phaser“, излучение медное, немонохроматизированное (оборудование федерального ЦКП „Материаловедение и диагностика в передовых технологиях“ (Bruker, Германия)).

3. С помощью измерителя иммитанса „Е7-20“ проводилось измерение электрической емкости конденсаторного элемента и тангенса диэлектрических потерь $(\operatorname{tg} \delta)$ на частоте $10 \mathrm{kHz}$ и измерительном поле $40 \mathrm{mV}$. Выбор данных параметров обусловлен хорошей воспроизводимостью получаемых результатов, а также тем, что пленка очень тонкая, и при больших полях может произойти пробой. Измеритель работает по методу „вольтметра-амперметра“. При приложении смещающих напряжений от 0 до $\pm 20 \mathrm{~V}$ погрешность измерения составляет $0.5 \%$. Значение диэлектрической проницаемости рассчитывалось по формуле: $\varepsilon=(C \cdot d) /\left(S \cdot \varepsilon_{0}\right)$, где $\varepsilon-$ эффективная величина диэлектрической проницаемости, $C-$ емкость плоского конденсатора, $d-$ толщина пленки, $S$ - площадь верхнего электрода, $\varepsilon_{0}-$ диэлектрическая постоянная.
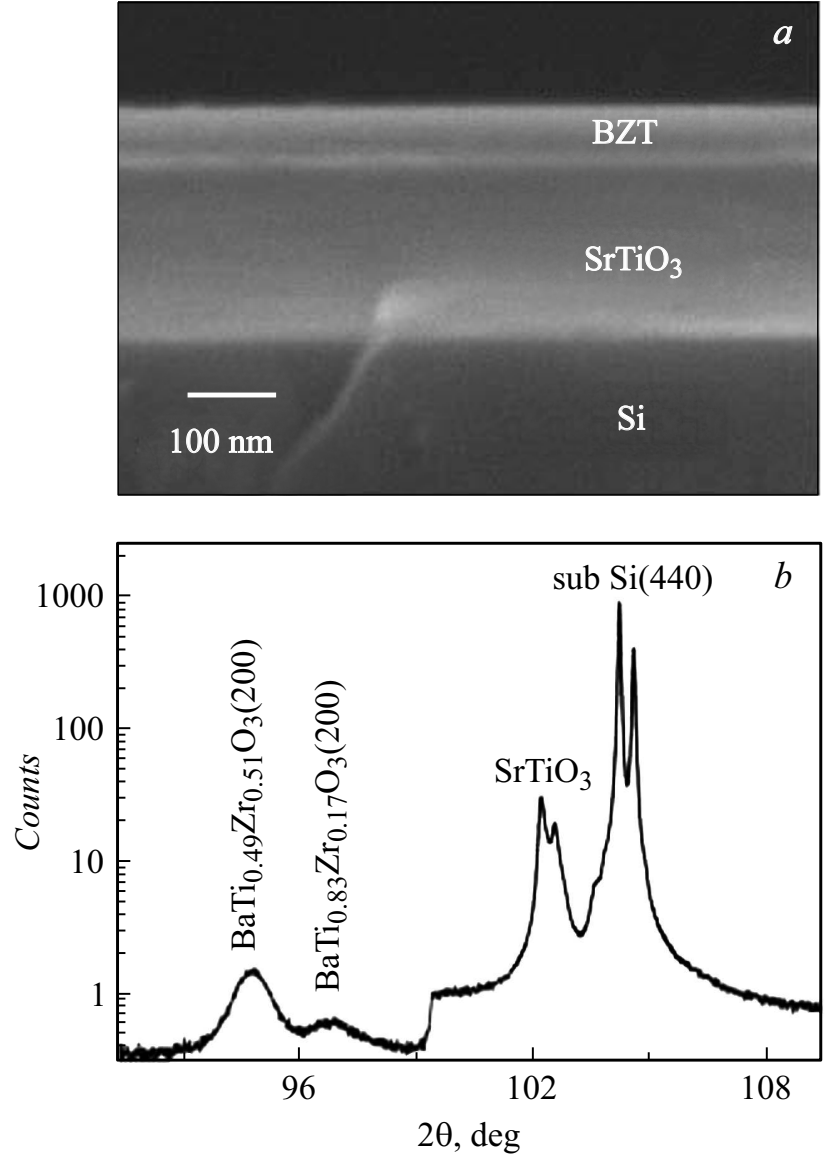

Рис. 1. Образец (\#2) $\mathrm{BaTi}_{0.65} \mathrm{Zr}_{0.35} \mathrm{O}_{3}(40 \mathrm{~nm}) / \mathrm{BaTi}_{0.8} \mathrm{Zr}_{0.2} \mathrm{O}_{3}$ $(20 \mathrm{~nm}) / \mathrm{BaTi}_{0.65} \mathrm{Zr}_{0.35} \mathrm{O}_{3}(40 \mathrm{~nm}): a-\mathrm{C} Э \mathrm{M} \mathrm{изображение,} b-$ рентгеновская дифракционная кривая.

\section{2. Обсуждение результатов}

С помощью сканирующей электронной микроскопии (СЭМ) были определены толщины получаемых BZTслоев. На рис. 1, а приведено СЭМ изображение для образца (\#2). Можно наблюдать следующие слои: подложка $\mathrm{Si}$, буферный слой $\mathrm{SrTiO}_{3}$ (толщиной $300 \mathrm{~nm}$ ) и составной BZT-слой. СЭМ изображения BZT-пленок не выявляют зерен или их границ. Данные рентгеновской дифрактометрии указывают на то, что слои BZT ориентированы по плоскости (100), что отличается от ориентации подложки, в то время как буферный слой имеет ту же ориентацию, что и подложка $\mathrm{Si}$ (110) (рис. $1, b$ ). Состав BZT в отношении концентраций Ti и $\mathrm{Zr}$ по данным дифрактометрии отвечает заявленным значениям. К сожалению, применяемая в настоящей работе установка не позволяет точно измерить упругие микронапряжения в исследуемых образцах из-за недостаточной степени монохроматичности излучения. Наблюдаемое уширение максимумов, отвечающих слоям BZT, обусловлено в основном малой наноразмерной толщиной исследуемых пленок (в образце (\#2) толщины слоев составляют $40 \mathrm{~nm} \mathrm{BaTi} 0_{0.65} \mathrm{Zr}_{0.35} \mathrm{O}_{3}, 20 \mathrm{~nm}$ 

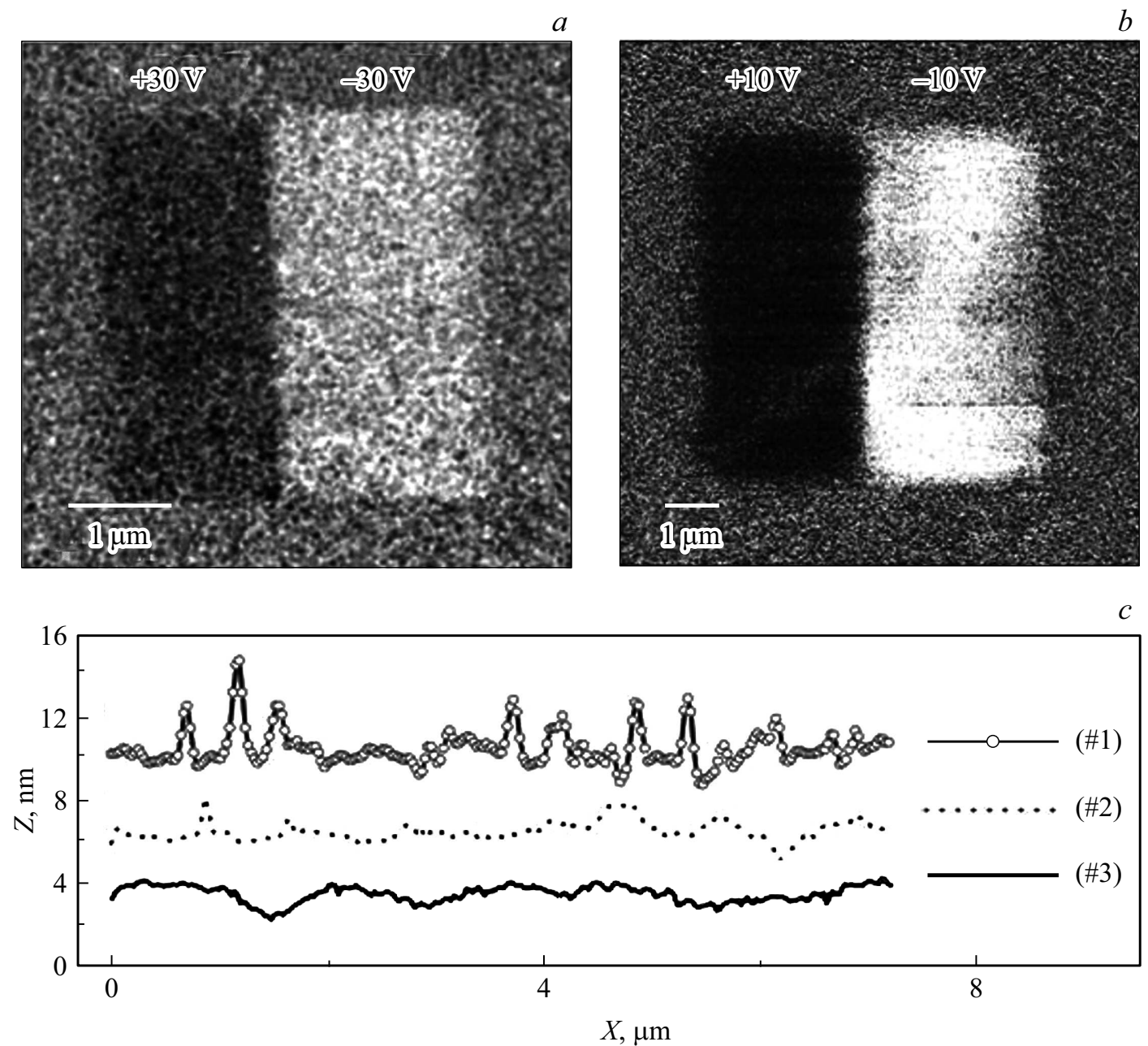

Рис. 2. $a-$ распределение сигнала пьезоотклика для образца (\#3); $b-$ карта протекания токов для образца (\#1). К темной области подавалось $+10 \mathrm{~V}$, к светлой $--10 \mathrm{~V} ; c-$ усредненные профили рельефа для всех образцов.

$\mathrm{BaTi}_{0.8} \mathrm{Zr}_{0.2} \mathrm{O}_{3}$ и $\left.40 \mathrm{~nm} \mathrm{BaTi}_{0.65} \mathrm{Zr}_{0.35} \mathrm{O}_{3}\right)$. Отчетливо видно, как различаются уширения пиков для тонкого $100 \mathrm{~nm}$ слоя BZT , буфера $\mathrm{SrTiO}_{3}$ (толщина $300 \mathrm{~nm}$ ) и подложки, которая оценивается как бесконечный кристалл. Толщины слоев, образующих пленку, согласовывались с данными СЭМ (рис. 1,a), а не с дифракционными данными (из-за нехватки монохроматичности излучения). Видно, что данные рентгеновской дифрактометрии и СЭМ согласуются, это позволяет нам предположить, что вклад микронапряжений в уширение линий не является существенным.

Для того чтобы детально исследовать процессы переполяризации указанных СЭ пленок, в настоящей работе применялся метод контактной С3М $[5,7,13]$. Эксперимент состоял из двух стадий:

1) „Поляризация“ - проводящий СЗМ-зонд приводится в контакт с поверхностью образца и к нему прикладывается положительное $\left(U_{+}=+10\right.$ и $\left.+30 \mathrm{~V}\right)$ либо отрицательное $\left(U_{-}=-10\right.$ и -30 напряжение. При этом переключение поляризации BZT-пленки в некоторой области под зондом. На этой стадии созда- вались протяженные поляризованные области размером $4 \times 2 \mu \mathrm{m}$;

2) „визуализация поляризованных областей“ - выполнялось сканирование области большей площади $(6 \times 6 \mu \mathrm{m})$, при этом на зонд подавалось малое напряжение $(2 \mathrm{~V})$ и регистрировался ток, протекающий сквозь пленку, либо сигнал пьезоэлектрического отклика (рис. $2, a)$.

На рис. 2, $а$ представлена карта распределения сигнала пьезоотклика для образца (\#3). Обнаружено, что переполяризовать данный образец возможно только при подаче существенных напряжений $( \pm 30 \mathrm{~V})$. В неполяризованных областях величина сигнала пьезоотклика мала, что может быть обусловлено тем, что поляризация в этих областях направлена вдоль поверхности.

На рис. 2,b представлена карта распределения токов в поляризованных областях для образца (\#1). Для образца (\#2) наблюдалось аналогичное распределение токов. Видно, что ток течет равномерно по всей поляризованной области, границ зерен не выявлено. Это указывает на отсутствие дополнительных каналов для 


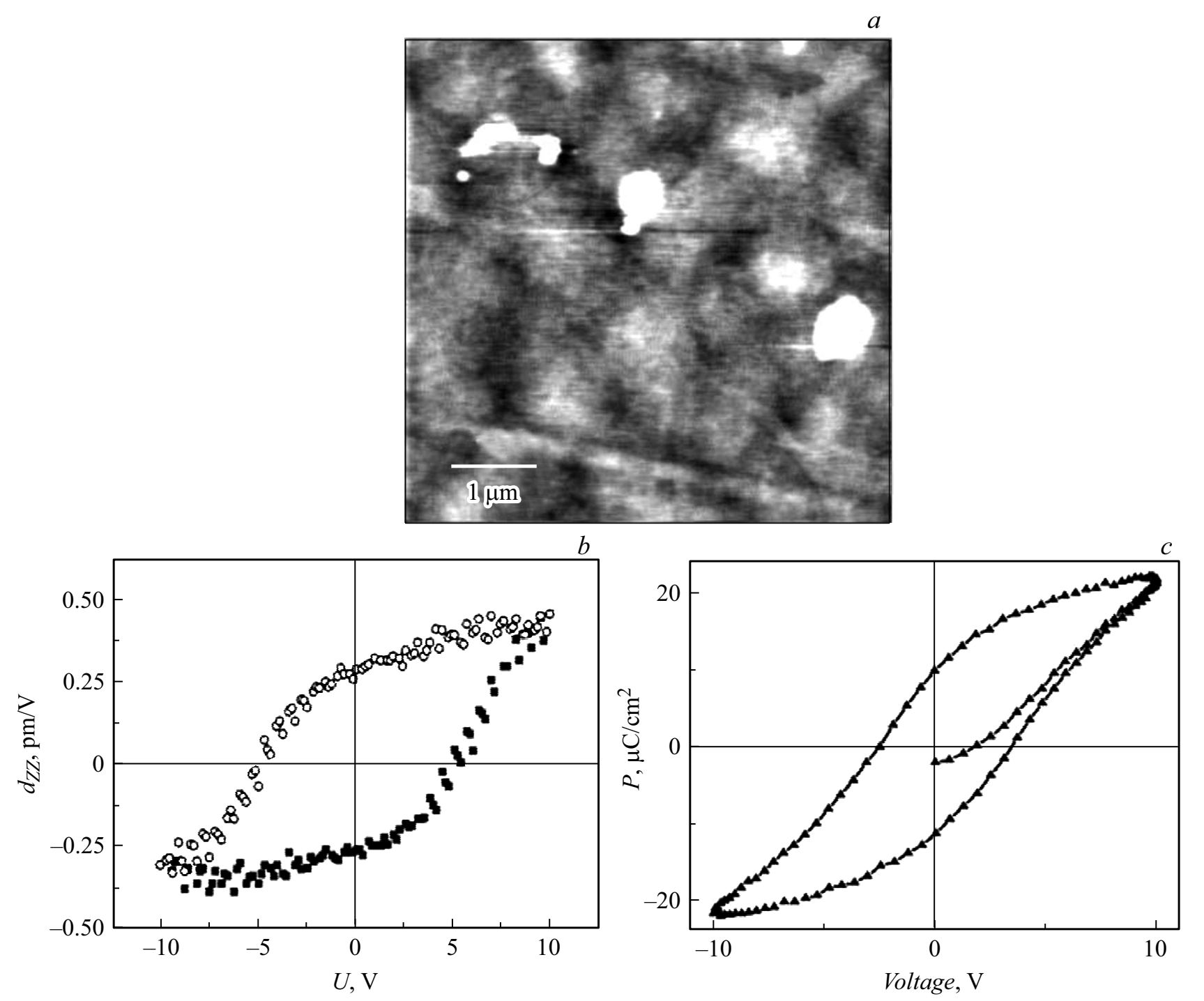

Рис. 3. Образец (\#3) $\mathrm{BaTi}_{0.8} \mathrm{Zr}_{0.2} \mathrm{O}_{3}: a-$ СЗМ топография; $b-$ петля гистерезиса, измеренная с помощью СЗМ, $c-$ петля гистерезиса, измеренная с помощью метода Сойера-Тауэра с электрода размером $200 \times 200 \mu \mathrm{m}$.

токов утечек, которыми обычно являются межзеренные границы $[5,14]$.

В „темных“ областях на рис. 2, $a, b$ вектор поляризации направлен от зонда в подложку (направление „сверху вниз“). В „светлых“ областях вектор поляризации направлен из подложки в зонд (направление „снизу вверх““). Экспериментально было установлено, что созданные поляризованные области могут сохраняться длительное время. В исследуемых пленках время жизни поляризованных областей составляло более $8 \mathrm{~h}$. Временная стабильность переполяризованных областей является важной характеристикой СЭ пленок, на базе которых может быть построена ячейка сегнетоэлектрической памяти.

Стоит отметить, что значение протекающих токов для образца (\#1) в областях, которые поляризованы „снизу вверх“, составляет $I_{+}=0.04 \mathrm{pA}$, а для состояния „сверху вниз“ $I_{-}=0.06 \mathrm{pA}$. Также обнаружено, что отноше- ние токов между направлениями „снизу вверх “ и „сверху вниз“ $I_{+} / I_{-}$различно для каждого образца. Так, для образцов (\#1) и (\#3) эта величина составляет $I_{+} / I_{-}=1.5$, а для образца (\#2) $I_{+} / I_{-}=2$. То есть проводимость в тонких BZT-пленках зависит от направления поляризации по отношению к приложенному внешнему полю. Этот эффект ранее наблюдался в РZТ-пленках [14]. Объяснение различий в величине токов может заключаться в перезарядке ловушечных центров, которые расположены в объеме пленки. В качестве таких центров также могут выступать кислородные вакансии [14]. Кроме того, установлено, что токи в BZT-пленках не являются емкостными переходными токами, которые были ранее обнаружены в PZT-пленках [14]. Надо отметить, что измерения токов в работе проводились с помощью СЗМ-зонда, площадь контактной области составляла $\sim 100 \mathrm{~nm}^{2}$, поэтому характерная величина плотностей протекающих токов в исследуемых образцах составляет 
$\sim 10^{-2} \mathrm{~A} / \mathrm{cm}^{2}$. Величины плотностей токов, измеренных в работе [15] для $\mathrm{BaTiO}_{3}$-пленок в низкопроводящем состоянии, составляют $\sim 10^{-1} \mathrm{~A} / \mathrm{cm}^{2}$. То есть добавление $\mathrm{Zr}$ позволяет на порядок снизить токи утечки в BZTструктурах. Стоит отметить, что характерные величины токов, протекающих в образцах (\#1)-(\#3), отличались несильно $\left(I_{\# 1} \approx 0.04-0.06 \mathrm{pA}, I_{\# 2,3} \approx 0.04-0.08 \mathrm{pA}\right)$.

Важным параметром исследуемых образцов является их гладкость. Наиболее гладким является образец (\#2), его шероховатость (RMS) составляет $0.2 \mathrm{~nm}$, для образца (\#3) $\mathrm{RMS}=0.3 \mathrm{~nm}$, для образца (\#1) $\mathrm{RMS} \sim 1 \mathrm{~nm}$ (рис. 2, $b$ ). Возможность роста достаточно гладких пленок открывает возможности для технологических применений тонких BZT-пленок в наноустройствах. Стоит отметить, что зернистость и шероховатость получаемых слоев BZT в зависимости от концентрации Zr исследовалась в ряде работ [16-18]. Так, в работе [16] средняя шероховатость образцов составляла $4 \mathrm{~nm}$ и зависимость от концентрации $\operatorname{Zr}_{x}(x=0.05,0.15,0.25)$ являлась нелинейной функцией и достигала наибольшего значения $4.5 \mathrm{~nm}$ при $x=0.15$. Уменьшение RMS при дальнейшем увеличении концентрации $\mathrm{Zr}$ связывалось с образованием более плотноупакованных и меньших по размеру зерен, а также с появлением фазы $\mathrm{ZrO}_{2}$. Аналогичная нелинейная зависимость $\mathrm{RMS}(\mathrm{x})$ была обнаружена в работе [18]. Характерные значения шероховатости в работе [18] составляли 4-5 nm, что было обусловлено увеличением постоянной решетки с увеличением концентрации $\mathrm{Zr}$, что приводило к уменьшению рассогласования решеток пленки и подложки, в результате чего вырастали большие по размеру зерна. Стоит отметить, что полученные в настоящей работе пленки гораздо более гладкие и обладают меньшей шероховатостью по сравнению с вышеперечисленными.

Измерение петель гистерезиса выполнялось в соответствии с методом, описанным в работе [13]. Для подачи коротких импульсов напряжения и регистрации сигнала пьезоэлектрического отклика пленки на это приложенное напряжение использовался специальный шаблон для растровой СЗМ литографии [13]. На рис. 3, $а$ представлена СЗМ топография (образец (\#3)), в центре которой была измерена петля гистерезиса локального пьезоэлектрического отклика (рис. $3, b$ ). Из данных рис. $3, b$ можно определить: а) коэффициент $d_{z z} \sim 1 \mathrm{pm} / \mathrm{V}$, б) величину коэрцитивного поля $E_{c}=U_{c} / d$, где $U_{c}-$ это коэрцитивное напряжение (около $5 \mathrm{~V}$ ), а $d$ - толщина пленки $(100 \mathrm{~nm})$. Величина измеренного коэффициента $d_{z z}$ оказалась более чем на порядок ниже, чем характерные значение пьезомодуля в пленке $\mathrm{BaTiO}_{3}$ $\left(d_{z z} \sim 50 \mathrm{pm} / \mathrm{V}\right)$ [19]. Стоит отметить, что измеряемое СЗМ зондом значение $d_{z z}$ оказывается в несколько раз заниженным, а $E_{c}$ завышенным из-за эффекта падения потенциала в приконтактной области зонд-поверхность. Кроме того, при концентрациях $Z r_{X}>0.2$ пленка BZT может (за счет понижения температуры Кюри) частично находиться в несегнетоэлектрической кубической фазе, что существенно снизит пьезоэлектрические свойства. Стоит отметить, что характерные значения $d_{z z}$ в образцах $(\# 1, \# 3)$ и $(\# 2)$ отличались не сильно $\left(d_{z z \# 1} \sim 0.5 \mathrm{pm} / \mathrm{V}, d_{z z \# 2} \sim 1 \mathrm{pm} / \mathrm{V}\right)$.

Измеренная в настоящей работе величина $E_{c}$ составляет $3 \cdot 10^{5} \mathrm{~V} / \mathrm{cm}$ и характерна для всех трех исследованных пленок. Это значение в несколько раз выше, чем для схожих по составу ВZT-пленок $E_{c}=0.5 \cdot 10^{5}[20]$ и $0.9 \cdot 10^{5} \mathrm{~V} / \mathrm{cm}[21]$. Авторы отмечают, что эта величина может быть связана с закреплением доменов микроструктурой и дефектами в пленке, а также зависеть от материала и ориентации подложки [22]. Стоит отметить, что измеренные с помощью СЗМ значения $E_{c}$ и $d_{z z}$ могут несколько отличаться от реальных значений. Это обусловлено следующим обстоятельством. При СЗМ измерениях приложенное к пленке напряжение распределено неравномерно. В области точечного контакта зонда с поверхностью возникает небольшая область, в которой может падать значительная часть (до 80\%) приложенного потенциала. Это приводит к тому, что измеренное значение $E_{c}$ оказывается завышенным в 5 раз, а значение $d_{z z}$ заниженным в 5 раз по сравнению с реальными величинами.

Для того чтобы определить величину остаточной поляризации, были проведены измерения петель диэлектрического гистерезиса $P-V$ (рис. 3,c) с макроскопического электрода размером $200 \times 200 \mu \mathrm{m}$. Измерения проводились стандартным методом Сойера-Тауэра с амплитудой синусоидального напряжения $10 \mathrm{~V}$ и частотой сигнала $64 \mathrm{~Hz}$. Величина остаточной поляризации составила $P_{r}=10 \mu \mathrm{C} / \mathrm{cm}^{2}$, что согласуется с характерными значениями остаточной поляризации в BZTпленках [23,24]. Петля гистерезиса не выходит на насыщение при напряжениях до $10 \mathrm{~V}$, что указывает на то, что при этом не все домены перевернулись. Это согласуется с представленными выше СЗМ данными, в которых показано, что полностью переключить поляризацию возможно подачей напряжения около $30 \mathrm{~V}$. Величины остаточной поляризации для разной полярности согласуются с симметричной величиной сигнала пьезоотклика для разных полярностей, измеренного с помощью СЗМ. Зависимость величины остаточной поляризации от температуры оценивалась авторами работы [23]. Величина $P_{r}$ составила от 22 до $15 \mu \mathrm{C} / \mathrm{cm}^{2}$ в диапазоне температур 300-400 К. Это изменение связывается с изменением внутренней структуры пленки и переходом ее от ромбической к кубической. В работе [24] изучалась зависимость величины $P_{r}$ от количества $\mathrm{Zr}$. Показано, что с увеличением концентрации $\mathrm{Zr}$ от $x=0$ до 0.35 происходит уменьшение величины остаточной поляризации с 8 до $5 \mu \mathrm{C} / \mathrm{cm}^{2}$. Такую зависимость авторы связывают с изменением размеров зерен. Чем больше размер зерен, тем больше доменные стенки и тем легче их переключить под действием внешнего электрического поля. Кроме того, авторы считают, что боковые механические взаимодействия между пленкой и подложкой приводят к двумерному эффекту зажима сегнетоэлектрических диполей, что также уменьшает 
величину $P_{r}$. Надо отметить, что для исследованных пленок с концентрацией $\mathrm{Zr}_{x=0.35}$ величина остаточной поляризации составила $P_{r}=15 \mu \mathrm{C} / \mathrm{cm}^{2}$, а для составной пленки -- сравнимое с представленным на рис. $3, c$. Таким образом, небольшие различия в величинах $P_{r}$ могут быть связаны и с материалом подложки, и с ориентацией пленок, и с размером зерен.

С помощью измерителя иммитанса „Е7-20“ были исследованы диэлектрические свойства BZT-пленок. Измерительное напряжение составляло $40 \mathrm{mV}$. Измеренная на частоте $10 \mathrm{kHz}$ величина диэлектрической проницаемости составила $\varepsilon \approx 120$, а тангенса диэлектрических потерь $\operatorname{tg} \delta \sim 0.02$. Измеренные значения величины диэлектрической проницаемости и диэлектрических потерь сопоставимы с измеренными в работах $[16,17,22,24]$.

\section{Заключение}

Таким образом, в работе показано, что BZT-пленки $(x=0.2,0.35)$ могут быть выращены монокристаллическими с достаточно гладкой поверхностью. RMSшероховатость для образца (\#2) с чередующимися слоями BZT20/BZT35/BZT20 составляет $0.2 \mathrm{~nm}$, для образца (\#3) $\left(\mathrm{Zr}_{x=0.2}\right) \mathrm{RMS}=0.3 \mathrm{~nm}$, для образца $(\# 1)$ $\left(\mathrm{Zr}_{x=0.35}\right) \mathrm{RMS} \sim 1 \mathrm{~nm}$. На образце (\#2) с чередующимися слоями наблюдаются крупные зерна шириной порядка $0.5 \mu \mathrm{m}$ и высотой $1-2 \mathrm{~nm}$. Величины измеряемых токов в исследуемых образцах достаточно малы $(\sim 0.06 \mathrm{pA})$, что свидетельствует о малых по сравнению с $\mathrm{BaTiO}_{3}$ утечках. Токи, протекающие вдоль областей, поляризованных „снизу вверх“ меньше, чем „сверху вниз“ и отличаются в 1.5 раза для однослойных образцов (\#1) и (\#3) и в 2 раза для гетероструктурного образца (\#2). Отсутствие межзеренных границ указывает на отсутствие каналов для дополнительных утечек тока. Об этом же свидетельствует малая величина измеряемого тангенса диэлектрических потерь ( $\operatorname{tg} \delta \sim 0.02)$. Малая характерная величина коэффициента пьезомодуля $d_{z z} \sim 1 \mathrm{pm} / \mathrm{V}$ указывает на возможное ослабление пьезоэлектрических свойств за счет снижения температуры Кюри при увеличении концентрации $\mathrm{Zr}$ и появления несегнетоэлектрической фазы.

\section{Финансирование работы}

Работа выполнена при поддержке гранта РФФИ № 18-32-00092_мол-а. Измерения методом сканирующей электронной микроскопии и рентгнодифракционные измерения выполнены с использованием оборудования федерального ЦКП „Материаловедение и диагностика в передовых технологиях“ (ФТИ им. А.Ф. Иоффе) при частичной финансовой поддержке Министерства образования и науки РФ (соглашение № 14.621.21.0013 от 28.08.2017, идентификатор проекта RFMEFI62117X0018)

\section{Конфликт интересов}

Авторы заявляют, что у них нет конфликта интересов.

\section{Список литературы}

[1] О.Г. Вендик. ФТТ, 51 (7), 1441 (2009). [O.G. Vendik. Phys. Solid State, 51 (7), 1529 (2009).]

[2] S. Gevorgian. Ferroelectrics in microwave devices, circuits and systems. (Springer, London, 2009), p. 396.

[3] T. Maiti, R. Guo, A.S. Bhalla. Appl. Phys. Lett. 89, 122909 (2006).

[4] W.S. Choi, B.S. Jang, D-G. Lim, J. Yi, B. Hong. J. Crystal Growth, 237-239, 438 (2002).

[5] Л.А. Делимова, Е.В. Гущина, Н.В. Зайцева, Д.С. Серегин, К.А. Воротилов, А.С. Сигов. ФТТ, 60 (3), 547 (2018). [L.A. Delimova, E.V. Gushchina, N.Z. Zaitseva, D.S. Seregin, K.A. Vorotilov, A.S. Sigov. Phys. Solid State, 60 (3), 553 (2018)].

[6] J. Qiana, P. Hu, Ch. Liu, J. Jiang, Zh. Dan, J. Ma, Y. Lin, Ce-Wen Nan, Y. Shen. Science Bulletin, 63 (6), 356 (2018).

[7] M. Kumari, D.G.B. Diestra, R. Katiyar, J. Shah, R.K. Kotnala, R. Chatterjee. J. Appl. Phys., 121, 034101 (2017).

[8] Q.R. Lin, D.Y. Wang, B.C. Luo, R. Ding, D.L. Lorenzen, S. Li. Appl. Surf. Sci., 331477 (2015).

[9] D. Wu, Ph. Sciau, S. Schamm, F. Gloux, M.V. Fernandez. J. Phys. D: Appl. Phys., 40, 4701 (2007).

[10] V.V. Shvartsman, D.C. Lupascu. J. Am. Ceram. Soc., 95, 1 (2012).

[11] S.J. Kuang, X.G. Tang, L.Y. Li, Y.P. Jiang, Q.X. Liu. Scripta Mater., 61, 68 (2009).

[12] W.F. Qin, J. Xiong, J. Zhu, J.L. Tang, W.J. Jie, Y. Zhang, Y.R. Li. J. Mater Sci., 43, 409 (2008).

[13] А.В. Анкудинов, А.Н. Титков. ФТТ, 47 (6), 1110 (2005). [A.V. Ankudinov, A.N. Titkov. Phys. Solid State, 47 (6), 1148 (2005).]

[14] Л.А. Делимова, Е.В. Гущина, В.С. Юферев, И.В. Грехов. ФTT, 56 (12), 2366 (2014). [L.A. Delimova, E.V. Gushchina, V.S. Yuferev, I.V. Grekhov. Phys. Solid State, 56 (12), 2451 (2014).]

[15] Ch.H. Jung, S.Ih. Woo. Thin Solid Films, 519, 3291 (2011).

[16] F.M. Pontes, M.T. Escote, C.C. Escudeiro, E.R. Leite, E. Londo. J. Appl. Phys., 96 (8), 4386 (2004).

[17] T.B. Wu, C.M. Wu, M.L. Chen. Appl. Phys. Lett., 69 (18), 2659 (1996).

[18] J.Z. Xin, C.W. Leung, H.L.W. Chan. Thin Solid Films, 519, 6313 (2011).

[19] V. Thery, Al. Bayart, J-F. Blach, P. Roussel, S. Saitzeka. Appl. Surf. Sci., 351, 480 (2015).

[20] W.J. Jie, J. Zhu, W.F. Qin, X.H. Wei, J. Xiong, Y. Zhang, A. Bhalla, Y.R. Li. J. Phys. D: Appl. Phys., 40, 2854 (2007).

[21] A.R.E. James, C. Prakash. Appl. Phys. Lett., 84, 1165 (2004).

[22] X.G. Tang, Q.X. Liu, Y.P. Jiang. J. Appl. Phys., 100, 114105 (2006).

[23] F. Guo, X. Wu, Qingshan Lu, Sh. Zhao. Ceramics International, 44, 2803 (2018).

[24] J. Zhai, X. Yao, J. Shen, L. Zhang, H. Chen. J. Phys. D: Appl. Phys., 37, 748 (2004). 